\title{
Editorials
}

\section{Making hospital discharge safer for frail older patients}

Hospital discharge represents one of the greatest challenges in interorganisational relationships in health care and can lay bare the potential failings of a healthcare system. The peri-discharge period is a risky time in a frail older patient's journey and clear communication is key to a safer discharge experience. Communication across the interface has been identified by the James Lind Alliance as one of the top three priorities for primary care patient safety. ${ }^{1}$ There are three settings (angles) for the people involved in discharge: hospital staff, primary/community care staff, and patients/carers who are going home - and all parties clearly want to communicate as effectively as possible. As there are three angles, this can be conceptualised as a communication triangle.

The burden of care in our healthcare system is widely acknowledged to have been shifting towards frail older patients. These patients have the highest risk of error and harm during care transitions ${ }^{2}$ because of multimorbidity, polypharmacy, and collapse of social networks. Research into discharge error and harm often focuses on this cohort though there are many other vulnerability factors, such as social deprivation, language barriers, and mental health problems. Safer discharge for an ever-increasing frail population against a background of increasing health inequality is complex, but may be addressed according to the angles of the communication triangle.

\section{THE HOSPITAL ANGLE}

Tensions are writ large in the literature related to intra- and interprofessional communication at the interface. Although GPs seldom report patient safety incidents related to discharge, when they do, communication problems are a key cause $(21 \%$ of reported incidents, $27 \%$ of which are related to the interface). ${ }^{3}$ The Royal College of Physicians, the Scottish Intercollegiate Guidelines Network, and the Professional Record Standards Body have done much to improve the quality of discharge summaries. ${ }^{4-6}$ Electronic discharge summary formats (from NHS Digital) are now mandatory. Despite substantial improvements to all aspects of discharge summary communication in the last 10 years, GPs continue to report issues with discharge summary communication: inappropriate delegation, unreasonable requests for investigations (both in terms of timeliness and appropriateness), unclear medication instructions, acronyms, and problems with clarity of responsibility for requested actions.? Hospitals have a fast turnover of patients, even for the complex frail caseload, which is thought to be one of the reasons for the high re-admission rate in this population. There is sometimes pressure to discharge to improve hospital flow or when the home environment is considered the safer option, for example, to reduce risk of nosocomial infection or prevent deconditioning. The most junior member of the hospital team must then produce a timely discharge summary.

Few healthcare systems have managed to cross the professional and structural/ organisational divide between primary and secondary care (though some, such as the Salford Integrated Record, have tried). Yet the changes to structures in primary care and organisation into networks provide an opportunity to allow primary care to communicate on a more even footing with secondary care. UK hospitals have scope to improve many elements of their pre-discharge work-up in order to reduce burden on primary care. 'Transitional care' (interventions that span the peri-discharge) is common in US hospitals. Most programmes focus on hospital-based services reaching out into the community, often reporting no difference in long-term outcomes despite high costs. ${ }^{8}$ One review suggests primary care-based transitional schemes might be more successful, but they have never been compared with secondary care-based interventions in head-to-head trials. ${ }^{8}$ Hospitals should be involved in borrowing elements of successful transition programmes that foster collaboration between primary and secondary care (such as Care Bridge, ${ }^{9}$ which reduced mortality within 6 months of discharge), perhaps focusing specifically on training their staff to educate patients about their time in hospital. Improving the discharge summary requires quality training for junior doctors, but a wider secondary care provider attitudinal shift is needed in relation to delegation of workload. GPs should be involved in discussion with their discharging hospitals about this issue and in the training of junior doctors on how to write discharge summaries. GPs should use primary care placements to train Foundation Year 2 doctors to handle discharge summaries at the primary care end.

\section{THE PRIMARY/COMMUNITY CARE ANGLE}

The author's epidemiological work estimated that $8 \%$ of a vulnerable cohort of $>75$-yearolds experienced harm associated with primary care management of discharge summaries. ${ }^{10}$ One example of high-severity harm was the need for a patient to have dialysis after incorrect post-discharge management of furosemide. What little is known about the chain of error shows that most problems happen at the point at which the GP reviews the summary; 7,10 in the case described above no arrangements were made for follow-up blood tests. More failures were found in relation to test ordering and instigating follow-up ( $25 \%$ of requests) than in medications management $117 \%$ of requests)..$^{10}$ We referred to these instances as 'failures' rather than errors because we cannot be sure about their causality. GPs certainly do not always agree with the requests made of them by secondary care, ${ }^{7}$ highlighting again the undercurrent of tension around 'delegation' of workload to primary care. There are known issues in primary care in relation to time and workload pressure that might lead to slips and lapses, but there are clearly other latent causes of error within primary care systems, such as lack of continuity between GP and patient or loopholes in investigation management systems.

Role diversification within general practice offers numerous options for involving alternative professionals in post-discharge management. The clinical pharmacist in the general practice scheme offers scope for pharmacists to be involved, but currently no data exist on what their impact might be in reducing error/harm related to medications reconciliation after discharge. ${ }^{11}$ Fragmentation of the discharge summary management process is potentially concerning, especially 
